\title{
IMAGING DIAGNOSIS-POSTMYELOGRAPHIC EPIDURAL LEAKAGE DUE TO INCREASED MENINGEAL PERMEABILITY
}

\author{
Ann Carstens, Adriaan M. Kitshoff
}

\section{History and Physical Findings}

The dog had acute pain in the hind quarters and a mild lameness in the right hind leg for $48 \mathrm{~h}$. When examined, there was loss of conscious proprioception, paralysis, and hyperreflexia of both hind legs, with loss of superficial and retention of deep pain. The panniculus reflex was absent at approximately the tenth thoracic vertebra (T10). There was some ataxia present in the forelimbs, but forelimb reflexes were normal as was the presence of superficial and deep pain. There was urine dribbling and increased ease in expressing the urinary bladder. The neurological examination was consistent with a spinal cord lesion between T10 and L6.

\section{Laboratory Findings}

Abnormal laboratory parameters were low red cell count at $5.21 \times 10^{12} / /$ (reference value of $5.5-8.521 \times$ $\left.10^{12} /\right)$ ), low hematocrit of 0.349 (reference value $0.37-0.55$ ) and mildly elevated white cell count of $18.5 \mathrm{x}$ $10^{9} /$ I (reference value $\left.6.0-15.0 \times 10^{9} / \mathrm{l}\right)$. The absolute mature neutrophil count was slightly elevated at 14.99 $\times 10^{9} /$ (reference value $3.0-11.5 \times 10^{9} /$ ), as was the absolute monocyte count at $1.85 \times 10^{9} / /$ (reference value $\left.0.15-1.35 \times 10^{9} / l\right)$.

\section{Imaging}

Survey spinal radiographs were normal. A myelogram was performed by placing a $22 \mathrm{G} 75 \mathrm{~mm}$ Terumo spinal needle into the subarachnoid space at L5/L6. Two milliliters of cerebrospinal fluid (CSF) was withdrawn, which was mildly blood contaminated. Nine milliliters of $300 \mathrm{mgl} / \mathrm{ml}$ iohexol was then injected into the subarachnoid space. In multiple views there was interrupted epidural leakage extending from the dorsal aspect of the subarachnoid space at the level of mid T12 caudally to the dorsal aspect of mid lumbar vertebra 2 (L2) (Fig. 1). Although this leakage did not communicate with the myelogram puncture site at L5/L6, it was initially thought to be iatrogenic. On the dorsoventral views a patchy contrast opacity was seen over the spinal cord from mid T10 to mid T13, hampering the evaluation. No signs of spinal cord compressive lesions were seen.

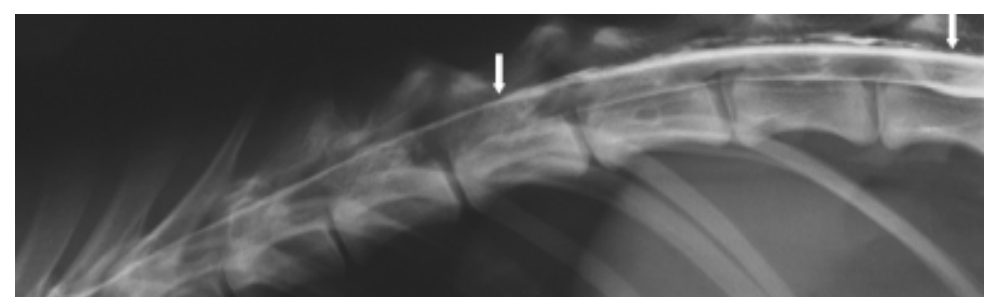

Fig. 1. Right lateral lumbar myelogram of thoracolumbar area. Note the epidural leakage extending from T12 caudally to L2 (arrows). This leakage did not communicate with the myelogram puncture site at L5/6. No signs of spinal cord compression were seen.

The next day the dog had no deep pain in the hind legs and there was progression of the forelimb ataxia. A followup cervical myelogram was performed to determine whether a compressive lesion cranial to T13 was 
present and to hopefully avoid epidural leakage allowing more thorough spinal cord evaluation. A $22 \mathrm{G} 75 \mathrm{~mm}$ spinal needle was placed into the subarachnoid space at the occipitoatlantal space. One milliliter of CSF was withdrawn and $12 \mathrm{ml}$ of $300 \mathrm{mgl} / \mathrm{ml}$ iohexol injected slowly. The same patchy contrast opacity overlying the spinal cord was seen from the mid to caudal thoracic region. Also, there was mild dorsal epidural leakage from T9 to mid T13 (Fig. 2). The dorsoventral view of the thoracolumbar area was difficult to evaluate due to the patchy overlying contrast medium. The radiologic diagnosis was caudal thoracic myelitis with secondary epidural leakage.

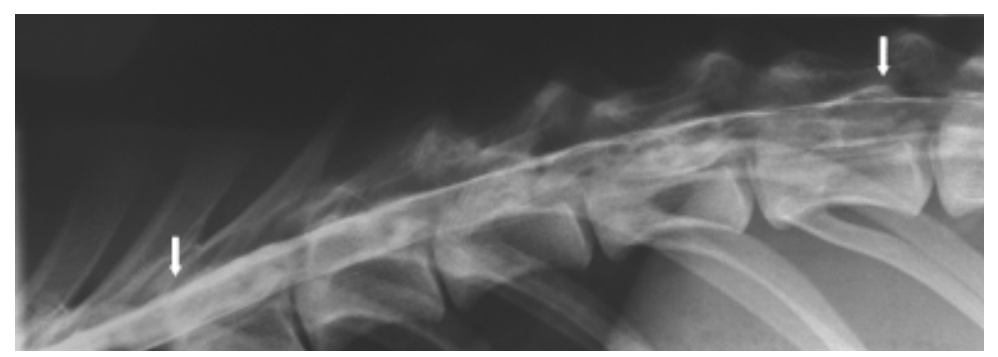

Fig. 2. Right lateral myelogram of the thoracolumbar area following occipito-atlantal injection. Note the patchy opacity superimposed over the spinal cord and evidence of mild dorsal epidural leakage from T9 to mid T13 (arrows).

\section{CSF Analysis}

\section{Lumbar Fluid}

The specific gravity was 1.007 (reference value of 1.030), and the protein value was $367.7 \mathrm{mg} / \mathrm{dl}$ (reference value $30-60 \mathrm{mg} / \mathrm{ml}$ ). The sample was very cellular with a red cell count of 528 cells/ $\mu$, a white cell count of $201 \mathrm{cells} / \mu \mathrm{l}$, and nucleated cells consisting of $22 \%$ neutrophils, 10\% lymphocytes, 12\% macrophages, and $56 \%$ eosinophils. The CSF fluid tested positive for canine distemper antibodies at a screening dilution of 1:10, Toxoplasma gondii was negative at $1: 10$ as was the Neospora caninum titer at 1:10.

\section{Cervical Fluid}

This fluid, which was inadequate in volume for a full-cell count had a specific gravity of $1.005,2 \%$ neutrophils, $24 \%$ lymphocytes, $72 \%$ active macrophages and $2 \%$ plasma cells. No eosinophils were seen. An indirect fluorescent antibody test for lgG antibodies was negative for canine distemper and canine parvovirus.

\section{Outcome}

Because of clinical deterioration despite symptomatic therapy, the absence of a specific diagnosis and a nonsurgical spinal cord lesion, the dog underwent euthanasia.

At a postmortem examination there was locally extensive hemorrhage of the spinal cord extending from T11 to T13 (Fig. 3). When sectioned transversely, there were multifocal petechiae and ecchymotic hemorrhage throughout the spinal cord from T11 to T13. Histologically the lesions consisted of necrosis and hemorrhage of the neuropil. Many blood vessels contained fibrinous thrombi and there was steatitis around some nerve roots. The dura mater had inflammatory changes with degenerated collagen outside the spinal cord attached to the dura. A specific etiology could not be found, although findings were consistent with trauma and/or an ischemic accident, and a diagnosis of idiopathic multifocal myelomalacia at T11-T13 and fibrinoid 
leptomeningeal ischemia was made. The ischemia was most likely responsible for increased permeability of the dura mater resulting in the leakage of the contrast medium epidurally.

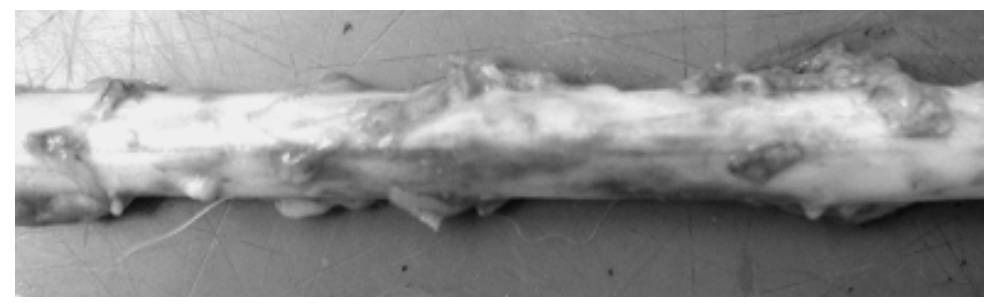

Fig. 3. Dorsum of the spinal cord from T11 to T13. Note the multifocal areas of hemorrhage as seen through the dura mater (cranial is to the left).

\section{Discussion}

Epidural leakage of contrast medium is usually as result of incorrect needle placement and occurs from the site of needle placement along the needle tract following the path of least resistance along the epidural space. ${ }^{1,2}$ The contrast medium then serves as a confounding opacity making it difficult or impossible to evaluate the subarachnoid space accurately. Epidural leakage due to meningeal pathology can also occur, and may be distant to the area of needle placement as described herein.

The epidural leakage distant to the site of myelographic injection in this dog was not iatrogenic because no communication was seen between the area of leakage and the site of contrast medium injection in either the lumbar or cervical myelogram. The leakage occurred spontaneously through the dura, as has been reported in humans. Spontaneous leakage can occur through dural tears at nerve roots caused by trauma, vigorous exercise or violent coughing; trauma initiating a dural tear need not be severe. ${ }^{3}$ Sneezing at high altitude has also led to dural tearing. ${ }^{4} \mathrm{~A}$ subarachnoid-pleural fistula developed in a dog after jumping and landing on her back. $^{5}$

The cause of the myelopathy in our dog is not known. A possible scenario was focal external trauma. Other causes may have been granulomatous meningoencephalitis ${ }^{6,7}$ or ischemia, as in fibrocartilaginous infarcts. ${ }^{8-}$

${ }^{10}$ Evidence of fibrocartilagenous infarction was not found, but cannot be excluded because vascular thrombi were found, although these may also have occurred secondary to trauma. The initial positive CSF titer for canine distemper was probably due to blood contamination because the second CSF sample was negative. The CSF eosinophilia in the first sample was also likely due to blood contamination.

Blunt trauma to the spinal cord can impair the blood-spinal cord barrier permeability to proteins leading to vasogenic edema which could lead to localized myelopathy. ${ }^{11}$ An investigation has been done to evaluate macromolecular transport structures that increased after the brain and spinal cord had been subjected to a variety of injuries. A theory was hypothesized that a unique endothelial cell system formed transendothelial cell channels defined as vesiculo-canalicular or vesiculo-tubular structures. These endothelial cell structures were seen in association with increased blood-brain barrier permeability of tracers, normally excluded from the intercellular milieu of the CNS. These structures appear to represent an anatomic gateway to the CNS, likely serving as conduits, but only became patent in damaged endothelial cells, and purportedly for inflammatory and neoplastic cells as well. ${ }^{12}$ If the dog in this report sustained trauma, increased leptomeningeal permeability could have allowed the extradural contrast medium leakage. Ischemia 
secondary to traumatically induced blood clot emboli leading to necrosis of the meninges could also have contributed to a break in the integrity of the meninges, in this case the dura mater, causing leakage of CSF. In conclusion, not all epidural contrast is iatrogenic and leptomeningeal leakage as a result of increased permeability should be suspected if the contrast medium leakage does not communicate with the needle tract.

\section{ACKNOWLEDGMENTS}

The authors thank Dr. I. Millward for providing the case and some diagnostic background, Prof. M. Williams for the pathological evaluation and Dr. Oglesby for additional insight.

\section{REFERENCES}

1. Kirberger RM. Recent developments in canine lumbar myelography. Comp Cont Educ Pract Vet 1994;16:847-854.

2. Kirberger RM, Wrigley RH. Myelography in the dog: review of patients with contrast medium in the central canal. Vet Radiol Ultrasound 1993;34:253-258.

3. Rando TA, Fishman R A. Spontaneous intracranial hypotension: report of two cases and review of the literature. Neurology 1992;42:481-487.

4. Spelle L, Boulin A, Tainturier C, Visot A, Graveleau P, Pierot L. Neuroimaging features of spontaneous intracranial hypotension. Neuroradiology 2001;43:622-627.

5. Packer RA, Frank PM, Chambers JN. Traumatic subarachnoidpleural fistula in a dog. Vet Radiol Ultrasound 2004;45:523-527.

6. Speciale J, Winkle TJ, Steinberg SA, Wortman JA. Computed tomography in the diagnosis of focal granulomatous meningoencephalitis: retrospective evaluation of three cases. J Am Anim Hosp Assoc 1992;28:327-332.

7. Glastonbury JRW, Frauenfelder AR. Granulomatous meningoencephalomyelitis in a dog. Aust Vet J 1981;57:186-189.

8. Junker K, Ingh TSGA, Bossard MM, Nes JJ. Fibrocartilaginous embolism of the spinal cord [FCE] in juvenile Irish Wolfhounds. Vet Quart 2000;22:154-156.

9. Hawthorne JC, Wallace LJ, Fenner WR, Waters DJ. Fibrocartilaginous embolic myelopathy in Miniature Schnauzers. J Am Anim Hosp Assoc 2001;37:374-383.

10. Widmer WR, Thrall DE. Canine and feline intervertebral disc disease, myelography, and spinal cord disease. In: Thrall DE (ed): Veterinary diagnostic radiology. Philadelphia: W.B.Saunders Co., 2002;110-126.

11. Sharma HS. Pathophysiology of blood-spinal cord barrier in traumatic injury and repair. Current Pharm Designs 2005;11:13531389.

12. Lossinsky AS, Shivers RR. Structural pathways for macromolecular and cellular transport across the blood-brain barrier during inflammatory conditions. Rev Hist Histopathol 2004;19:535-564. 